\title{
Disentangling geometric and dissipative origins of negative Casimir entropies
}

\author{
Stefan Umrath, ${ }^{1, *}$ Michael Hartmann, ${ }^{1}$ Gert-Ludwig Ingold, ${ }^{1, \dagger}$ and Paulo A. Maia Neto ${ }^{2}$ \\ ${ }^{1}$ Institut für Physik, Universität Augsburg, Universitätsstraße 1, D-86135 Augsburg, Germany \\ ${ }^{2}$ Instituto de Física, UFRJ, CP 68528, Rio de Janeiro RJ 21941-909, Brazil
}

(Received 21 July 2015; published 12 October 2015)

\begin{abstract}
Dissipative electromagnetic response and scattering geometry are potential sources for the appearance of a negative Casimir entropy. We show that the dissipative contribution familiar from the plane-plane geometry appears also in the plane-sphere and the sphere-sphere geometries and adds to the negative Casimir entropy known to exist in these geometries even for perfectly reflecting objects. Taking the sphere-sphere geometry as an example, we carry out a scattering-channel analysis, which allows us to distinguish between the contributions of different polarizations. We demonstrate that dissipation and geometry share a common feature making possible negative values of the Casimir entropy. In both cases there exists a scattering channel whose contribution to the Casimir free energy vanishes in the high-temperature limit. While the mode-mixing channel is associated with the geometric origin, the transverse electric channel is associated with the dissipative origin of the negative Casimir entropy. By going beyond the Rayleigh limit, we find even for large distances that negative Casimir entropies can occur also for Drude-type metals provided the dissipation strength is sufficiently small.
\end{abstract}

DOI: 10.1103/PhysRevE.92.042125

PACS number(s): 05.70.Ce, 42.50.Lc, 03.65.Yz

\section{INTRODUCTION}

The specific heat of a system coupled infinitely weakly to a thermal heat bath always has to be positive. This statement derives directly from the fact that the specific heat is proportional to the variance of the system energy. By appropriately integrating the specific heat, it follows that also the entropy has to be positive. Nevertheless, there are a number of situations in which the existence of a negative specific heat or a negative entropy is discussed.

Such a situation immediately arises when the coupling between the system and the heat bath is no longer infinitely weak [1,2]. Then, the specific heat of the system can be defined as the difference between the specific heat of the system plus heat bath on the one hand and the specific heat of the heat bath alone on the other hand. While there is no reason why the difference of two positive numbers has to be positive, one would typically expect that coupling additional degrees of freedom will increase the specific heat. The difference of specific heats just introduced is therefore expected to be positive. The same reasoning applies to the entropy.

A notable exception is the damped free particle, for which under appropriate circumstances, the specific heat can become negative [1,3-5]. An interesting feature of this system consists in the fact that its properties depend on the dimensionless ratio $k_{B} T / \hbar \gamma$, where $T$ is the temperature and $\gamma$ the damping constant, while $k_{B}$ and $\hbar$ are the Boltzmann constant and the Planck constant, respectively. As a consequence, and in contrast to common expectations, decreasing the damping constant $\gamma$ will render the free particle more classical and shift the transition to the quantum behavior to lower temperatures. A negative specific heat in the sense just discussed is also found in certain systems in condensed-matter physics and related fields [6-10].

\footnotetext{
*stefan.umrath@physik.uni-augsburg.de

${ }^{\dagger}$ gert.ingold@physik.uni-augsburg.de
}

Another important case where negative entropies give rise to considerable interest is the Casimir effect, which we will consider in more detail in the present paper. Even though experimentally the Casimir force is much more relevant than the Casimir entropy, the former contains a significant zerotemperature contribution while the latter allows us to focus on the effect of thermal photons, thereby highlighting nontrivial aspects of quantum statistical mechanics.

Suppose that we consider the Casimir interaction between two objects. With each of the objects as well as with the combination of the two objects we can associate an entropy as the difference between the entropy of the electromagnetic field in the presence of the object(s) and the entropy of the free electromagnetic field. The Casimir entropy is an interaction entropy, which then is obtained by subtracting the entropies of the two individual objects from the entropy of the two objects. While individual entropies are not finite, the Casimir entropy yields a finite value.

One potential source of a negative Casimir entropy is the coupling of the electromagnetic field to the electrons in the scattering objects. These electrons undergo a dissipative motion and therefore the electromagnetic response of the objects is characterized by a damping constant $\gamma$. The presence of dissipation inside the scatterers leads to a suppressed reflectivity of transverse electric (TE) modes at low frequencies while the scattering of transverse magnetic (TM) modes is not affected significantly. As a consequence, in the plane-plane geometry a reduction of the Casimir force [11] and Casimir entropy by a factor of two is found at high temperatures. The possibility of a negative Casimir entropy has been the subject of an extensive debate over more than a decade [12-30].

A thorough understanding of the role of dissipation in the Casimir effect is of considerable interest for the interpretation of experiments. Presently, there still exists disagreement about whether the dissipative low-frequency part of the electromagnetic response is relevant or not. While some experiments are interpreted in terms of the dissipation-less plasma model [31,32], other experiments are interpreted in terms of the Drude model, which includes dissipation $[33,34]$. At this point, it is 
useful to recall that a nonvanishing value of $\gamma$ is required for the Lifshitz formula [35] to be applicable, as shown explicitly by analyzing the properties of the electromagnetic modes in the complex plane and their contributions to the radiation pressure [36].

Most Casimir force measurements are carried out in the plane-sphere geometry with a surface-to-surface distance between 0.1 and a few micrometers. Depending on the experiment, the radius of the sphere or the radius of curvature of a spherical segment ranges from $20 \mu \mathrm{m}$ [37,38] to about $20 \mathrm{~cm}$ [39]. The latter case is rather close to a configuration of two parallel plates, where TE and TM modes do not mix. However, with decreasing sphere radius, polarization mixing becomes increasingly relevant and it is important to understand how the occurrence of negative Casimir entropies depends on dissipation in the plane-sphere and sphere-sphere geometry.

The extension of the discussion of the negative Casimir entropy due to dissipation beyond the plane-plane geometry is not the only purpose of this paper. In fact, it has been known for some time that negative Casimir entropies can arise for the plane-sphere [23,24,26] and the sphere-sphere [28] geometry, even for perfect conductors. An important question, therefore, concerns the relative status of the negative Casimir entropies due to dissipation on the one hand and due to geometry on the other hand.

Recently, it was shown that the negative Casimir entropy in the perfect metal plane-sphere and sphere-sphere geometries can be traced back to the mixing between the TE and TM modes of the electromagnetic field [29,30]. This identification became possible by realizing that negative Casimir entropies are most pronounced at large distances between the scattering objects. Then, within a scattering formalism, it is sufficient to consider one round-trip of electromagnetic waves between the scatterers and a decomposition into the various scattering channels becomes possible.

In Refs. [29,30] it was argued that in the large-distance limit two Drude-type metal objects will not allow for a negative Casimir entropy because the reflection of the TE modes is strongly suppressed and only a positive contribution of TM modes is left. This regime can be attained for any given damping strength by making the distance between the scattering objects sufficiently large. On the other hand, for any given distance of the objects, one can, at least in principle, make the damping strength so small that a negative Casimir entropy ensues.

In order to disentangle the contributions to a negative Casimir entropy arising from dissipation and geometry, we will go beyond the electric dipole approximation, i.e., the Rayleigh limit [40] employed in Ref. [29]. While we will assume the distance $d$ between the scattering objects to be large compared to the radii of the spherical objects involved, we will allow for damping strengths so small that a dissipative contribution to the negative Casimir entropy can occur in addition to the geometric contribution.

In Sec. II, we start by sketching the formalism required for the polarization channel analysis introduced in Ref. [29]. By considering the Casimir entropy for the plane-plane, sphere-plane, and sphere-sphere geometries, we then illustrate how negative Casimir entropies of dissipative and geometric origin manifest themselves in the temperature dependence of the Casimir entropy. In Sec. III, the contributions from the various scattering channels are analyzed within the dipole approximation but beyond the Rayleigh limit. The approximation of a single scattering round-trip between the two scattering objects allows us to disentangle dissipation and geometry as sources of a negative Casimir entropy and at the same time to identify common features. In Sec. IV, we go beyond the single round-trip approximation and demonstrate that repeated scattering round-trips and, for perfect conductors, higher multipoles tend to suppress negative Casimir entropies. In Sec. V we present our conclusions. An appendix collects some formulas needed for the evaluation of the Casimir entropy within the scattering formalism.

\section{DISSIPATIVE AND GEOMETRICAL INFLUENCE ON CASIMIR THERMODYNAMICS}

The Casimir entropy is obtained from the Casimir free energy $\mathcal{F}$ by means of the usual thermodynamic relation,

$$
\mathcal{S}=-\frac{\partial \mathcal{F}}{\partial T}
$$

Within the multiple-scattering theory [41-44], the Casimir free energy can be expressed as

$$
\mathcal{F}=\frac{k_{B} T}{2} \sum_{n=-\infty}^{\infty} \operatorname{Tr}\left(\ln \left[1-\mathcal{M}\left(\left|\xi_{n}\right|\right)\right]\right),
$$

with the Matsubara frequencies $\xi_{n}=2 \pi n k_{B} T / \hbar$. The matrix $\mathcal{M}$ describes round-trips of electromagnetic waves between all involved scatterers at imaginary frequencies $i \xi_{n}$.

As we will focus on the sphere-sphere and plane-sphere geometries, the trace in Eq. (2) can be taken in a spherical wave basis with the angular part given by spherical harmonics of degree $\ell$ and order $m$. In view of the axial symmetry of the geometries considered here, $m$ is conserved during the scattering process. The round-trip operator in a corresponding subspace,

$$
\mathcal{M}^{(m)}=\mathcal{R}_{1}^{(m)} \mathcal{T}_{12}^{(m)} \mathcal{R}_{2}^{(m)} \mathcal{T}_{21}^{(m)}
$$

can be expressed as product of translation operators $\mathcal{T}_{i j}$ from the reference frame of object $j$ to that of object $i$ and reflection operators $\mathcal{R}_{i}$ associated with object $i$. For reference, explicit expressions for the sphere-sphere geometry involving Mie coefficients and spherical wave translation formulas are given in the Appendix.

For large distances between the scattering objects, all matrix elements of the round-trip operator $\mathcal{M}$ will be much smaller than one. The Casimir free energy Eq. (2) can then be linearized,

$$
\mathcal{F} \approx-\frac{k_{B} T}{2} \sum_{n=-\infty}^{\infty} \sum_{m=-\infty}^{\infty} \operatorname{Tr} \mathcal{M}^{(|m|)}\left(\left|\xi_{n}\right|\right),
$$

thereby retaining only contributions from single round-trips. Within this approximation it is possible to decompose the Casimir free energy $\mathcal{F}$ and the Casimir entropy $\mathcal{S}$ into contributions from the different scattering channels and thereby to gain physical insight $[29,30]$. Of particular interest will be the channel where the TE polarization is conserved during the 
round-trip and the polarization-mixing channel where each of the two objects scatters a different polarization.

Furthermore, for sufficiently large separations $d / R \gtrsim 20$, the dipole approximation applies to the Casimir entropy and we can restrict ourselves to $\ell=1$ and $|m|=0,1$ (see Figs. 5 and 7 below). We will make use of the single round-trip dipole approximation in the present section as well as in the following Sec. III. Its range of validity will be discussed in Sec. IV.

In order to obtain a first idea of the interplay between the dissipative and geometric contributions to the negative Casimir entropy in the plane-sphere and sphere-sphere geometries, we compare with the plane-plane geometry where the negative Casimir entropy is solely of dissipative origin. Apart from the special case of perfectly conducting metals $(\mathrm{P})$, we will consider nonmagnetic Drude-type (D) metals with the frequency-dependent permittivity,

$$
\varepsilon(\omega)=1-\frac{\omega_{P}^{2}}{\omega(\omega+i \gamma)} .
$$

Here, $\gamma$ and $\omega_{P}$ are the damping constant and the plasma frequency, respectively, corresponding to a dc conductivity $\sigma_{0}=\omega_{P}^{2} / \gamma$. While $\omega_{P}$ determines the frequency scale beyond which the metal becomes transparent, a finite value of $\sigma_{0}$ results in a vanishing reflectivity of the TE modes at zero frequency.

Figure 1 shows the temperature dependence of the Casimir entropy for the three geometries indicated by the insets. The entropy is scaled by its high-temperature value $\mathcal{S}_{\mathrm{HT}}^{\mathrm{P}}$ for perfectly conducting metals. A dimensionless temperature is defined by means of the distance $d$ between the reference frames of the two scattering objects. The three solid lines correspond to Drude-type metals with $\gamma d / c=10^{-2}, 10^{2}$, and $10^{4}$ increasing from bottom to top and $\omega_{P} d / 2 \pi c=400$. Here, $c$ is the speed of light. The lower (blue) curve and the upper (red) curve thus correspond to good and bad conductors, respectively. As a reference, the case of a perfectly conducting metal is depicted as dashed line.

We start by recalling in Fig. 1(a) the main features of the Casimir entropy for Drude-type metals in the plane-plane geometry. It is well known that a finite dc conductivity suppresses the zero-frequency contribution of the TE modes, resulting in a reduction $\Delta \mathcal{S}_{\mathrm{HT}}^{\mathrm{TE}}$ of the Casimir entropy at high temperatures by a factor of two with respect to the case of an infinite dc conductivity [11]. The arrows in Fig. 1 visualize the effect of this missing zero-frequency contribution to the Casimir entropy.

Since for perfectly conducting metals the Casimir entropy decreases with decreasing temperature, the missing zerofrequency contribution of the TE modes exceeds their contribution at finite temperatures. As a consequence, at sufficiently low temperatures, the Casimir entropy for Drude-type metals becomes negative, provided the dc conductivity is sufficiently large. For the smallest value of the damping constant $\gamma$ in Fig. 1(a) the entropy curve over a large temperature range resembles closely that for a perfect conductor except for the entropy shift $\Delta \mathcal{S}_{\mathrm{HT}}^{\mathrm{TE}}$.

The Casimir entropy approaches its vanishing zerotemperature value only when the temperature falls below a temperature of the order of $\hbar \gamma / k_{B}$ [21]. The middle (black) curve therefore comes close to zero at much higher
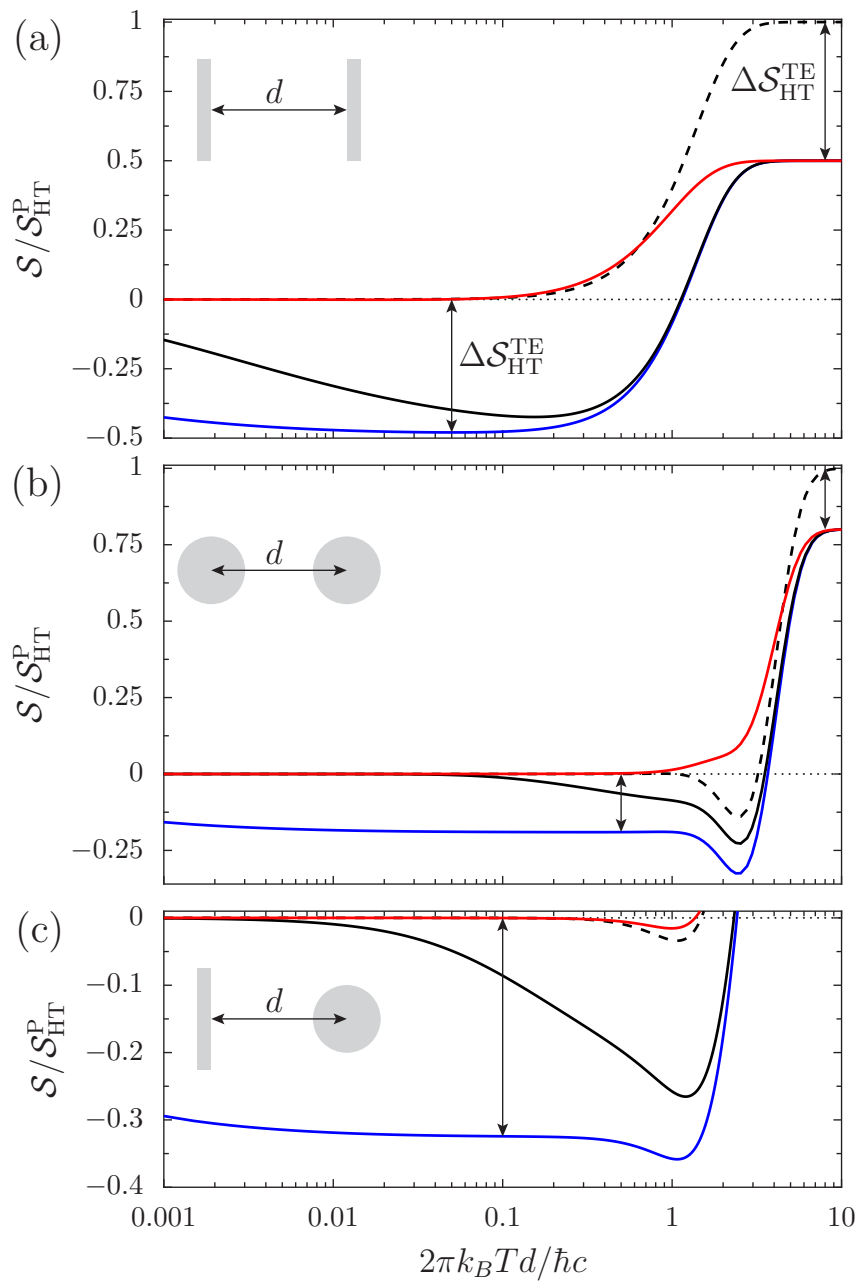

FIG. 1. (Color online) The Casimir entropy $\mathcal{S}$ scaled by its hightemperature value $\mathcal{S}_{\mathrm{HT}}^{\mathrm{P}}$ for perfect conductors is shown as a function of temperature for the (a) plane-plane geometry, (b) sphere-sphere geometry, and (c) plane-sphere geometry. While the dashed lines represent the entropy for perfectly conducting objects, the solid lines correspond to Drude-type conductors with the damping constant $\gamma d / c=10^{-2}, 10^{2}$, and $10^{4}$ increasing from bottom to top and the plasma frequency given by $\omega_{P} d / 2 \pi c=400$. The sphere radii are chosen as $R=d / 20$. The arrows indicate the effect of the missing zero-frequency contribution to the Casimir entropy for Drude-type metals with respect to perfect conductors.

temperatures than the lower (blue) curve. This behavior is reminiscent of the damped free particle discussed in the introduction where the temperature scale is also set by the damping constant $\gamma$.

A reduction of the Casimir entropy at high temperatures as well as negative values at lower temperatures with a crossover to vanishing Casimir entropy on a temperature scale given by $\gamma$ are also visible for the sphere-sphere geometry in Fig. 1(b) and the plane-sphere geometry in Fig. 1(c). Since the two geometries share the same qualitative features, we restrict the latter figure to negative values of the Casimir entropy. In both figures, the sphere radii are chosen as $R=d / 20$ to ensure the validity of the single round-trip dipole approximation. 
There are quantitative differences in the temperature dependence of the Casimir entropy for the three geometries. While the temperature scale on which the Casimir entropy approaches zero at low temperatures clearly increases with increasing damping constant $\gamma$, its value depends on the scattering geometry. In addition, the reduction of the Casimir entropy at high temperatures amounts only to $1 / 3$ for the plane-sphere geometry and $1 / 5$ for the sphere-sphere geometry instead of $1 / 2$ for the plane-plane geometry. This reduction is a consequence of the fact that for a perfectly conducting sphere the reflectivity of the TE modes is only one half of the reflectivity of the TM modes.

Besides the features already present in the plane-plane geometry, an additional dip in the Casimir entropy appears for the sphere-sphere and plane-sphere geometries at temperatures where $2 \pi k_{B} T d / \hbar c$ is of order one. This feature is of geometric origin and survives even in the absence of dissipation as can be seen from the dashed lines in Figs. 1(b) and 1(c). Its position depends on the geometry and it is more pronounced in the sphere-sphere geometry.

While for a damping constant as large as $\gamma=10^{4} c / d$, i.e., for the upper (red) solid curves, the Casimir entropy still becomes negative in the plane-sphere geometry, it remains positive for all temperatures in the sphere-sphere geometry. Nevertheless, even there a remnant of the dip is visible. However, for sufficiently large damping constant $\gamma$, the contribution to the Casimir entropy involving TE modes will disappear [29,30]. For such strong damping, the electrons will suffer many collisions during an oscillation period of the electromagnetic field for all relevant frequencies. The total irradiating power in the TE modes will then be dissipated, thereby suppressing the reflection of these modes.

\section{CHANNEL ANALYSIS BEYOND THE RAYLEIGH LIMIT}

The temperature dependence of the Casimir entropy displayed in Figs. 1(b) and 1(c) suggests that the negative Casimir entropy can be separated into contributions of dissipative and of geometric origin. This is particularly clear for the spheresphere geometry for which we will demonstrate in the second half of this section that the expected separation is indeed possible in terms of different scattering channels. Even though we will be able to disentangle geometric and dissipative parts, Figs. 1(b) and 1(c) also show that the geometric contribution depends on the damping constant appearing in the Drude-type permittivity Eq. (5). In order to analyze this dependence, we will need to take a closer look at the scattering properties of Drude-type metal spheres.

For the sphere-sphere geometry, the translation operators $\mathcal{T}$ appearing in Eq. (3) restrict the relevant wave numbers to small values with $k d \lesssim 1$. This cutoff is caused by the modified spherical Bessel functions present in Eq. (A4). For large distances $d \gg R$, one may therefore apply the Rayleigh limit, where only dipole scattering is relevant. For perfect metal spheres, it will be sufficient to consider only the leading term in the expansion of the $\ell=1$ Mie coefficients for TM modes,

$$
a_{1}^{\mathrm{P}}=-\frac{2}{3}(k R)^{3}+\frac{1}{5}(k R)^{5}+\mathcal{O}\left(k^{6}\right),
$$

as well as for TE modes

$$
b_{1}^{\mathrm{P}}=\frac{1}{3}(k R)^{3}+\frac{1}{5}(k R)^{5}+\mathcal{O}\left(k^{6}\right) .
$$

Here, TM and TE scattering amplitudes are of the same order with a relative factor of two alluded to in the previous section.

In contrast, for Drude-type metal spheres, the Mie coefficients for TM modes with $\ell=1$,

$$
a_{1}^{\mathrm{D}}=-\frac{2}{3}(k R)^{3}+\frac{2 c}{\sigma_{0} R}(k R)^{4}+\mathcal{O}\left(k^{5}\right),
$$

and for TE modes,

$b_{1}^{\mathrm{D}}=\frac{R \sigma_{0}}{45 c}(k R)^{4}-\frac{1}{45}\left[\frac{2}{21}\left(\frac{\sigma_{0} R}{c}\right)^{2}+\frac{\sigma_{0}}{\gamma}\right](k R)^{5}+\mathcal{O}\left(k^{6}\right)$,

differ in the power of $k R$ in the leading term. The scattering of TE modes thus becomes negligible in the Rayleigh limit. However, the prefactor of the leading term in Eq. (9) indicates the appearance of a new scale. As the comparison of Eqs. (8) and (9) shows, the scattering of TE modes can only be neglected if

$$
\frac{R \sigma_{0}}{30 c} \ll \frac{d}{R} .
$$

The applicability of the Rayleigh limit thus also depends on the dc conductivity $\sigma_{0}$. For a given de conductivity, it is always possible to reach the large-distance limit where the scattering of TE modes can be neglected. On the other hand, for a given distance, the Rayleigh limit ceases to hold if the dc conductivity becomes too large.

A physical interpretation of the condition Eq. (10) can be given in terms of the quasistatic skin depth,

$$
\delta(\omega)=\left(\frac{2 c^{2}}{\sigma_{0} \omega}\right)^{1 / 2} .
$$

Scattering of TE modes can thus only be neglected provided the sphere is sufficiently small compared to the skin depth,

$$
R \ll \sqrt{15} \delta,
$$

in the relevant frequency range. Then, no eddy currents can be induced and the sphere becomes transparent to the TE modes.

The breakdown of the validity of the Rayleigh regime for a well conducting metal sphere with $\sigma_{0} R / c=4 \pi \times 10^{4}$ is illustrated in Fig. 2. The ratio of Mie coefficients $-b_{1}^{\mathrm{D}} / a_{1}^{\mathrm{D}}$ for dipole scattering approaches at small wave numbers the behavior predicted by the leading terms in Eqs. (8) and (9). At larger wave numbers, the dipole regime is entered where the ratio of the Mie coefficients $a_{1}^{\mathrm{D}}$ and $b_{1}^{\mathrm{D}}$ approaches the value for perfectly conducting spheres given by Eqs. (6) and (7). Increasing the wave numbers even further, the multipole regime is reached where first the scattering of TM modes with $\ell=2$ described by the Mie coefficient $a_{2}^{\mathrm{D}}$ becomes relevant.

An increase in the dc conductivity will reduce the Rayleigh regime and render the dipole regime valid at even smaller frequencies. To ensure the validity of the Rayleigh limit, one thus has to choose either bad conductors or go to large distances satisfying Eq. (10). In the large-distance limit considered in Refs. [29] and [30], it was assumed that Eq. (10) holds and that 


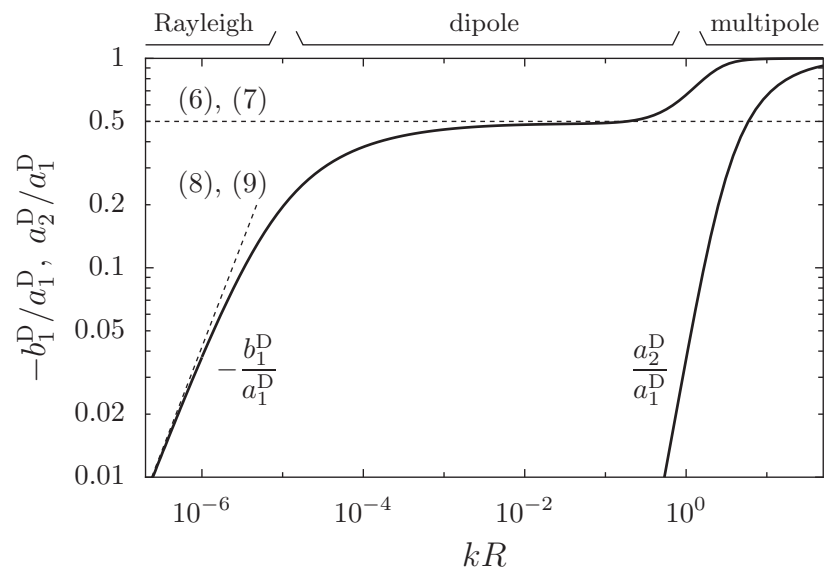

FIG. 2. The ratios of Mie coefficients $-b_{1}^{\mathrm{D}} / a_{1}^{\mathrm{D}}$ and $a_{2}^{\mathrm{D}} / a_{1}^{\mathrm{D}}$ as a function of the wave number $k$ are shown for a Drude-type metal sphere with radius $R$. The plasma frequency and the damping constant are given by $\omega_{P} R / 2 \pi c=20$ and $\gamma / \omega_{P}=10^{-4}$. Three different regimes can be distinguished. In the Rayleigh regime, TE scattering is negligible, while in the dipole regime, TE and TM scattering are of comparable strength. In the multipole regime, the scattering of higher multipole waves becomes relevant. The dashed lines indicate the approximations valid in the first two regimes with references to the corresponding equations.

the TE modes are not scattered by Drude-type metal spheres. Under this condition, it was possible to derive analytical results for the Casimir entropy. Here, we will allow for an arbitrary dc conductivity and thus have to resort to a numerical evaluation of the Casimir entropy employing the full Mie coefficients given in the Appendix.

In the large-distance limit $d \gg R$, where the linearized single round-trip expression for the Casimir free energy [cf. Eq. (4)] applies, we can decompose the Casimir entropy into contributions arising from the scattering channels characterized by the polarization on the two spheres. For two channels the polarization on the spheres is conserved along the round-trip: $\mathrm{TM} \leftrightarrows \mathrm{TM}$ and $\mathrm{TE} \leftrightarrows \mathrm{TE}$. In the following, we will refer to them as TM and TE channels, respectively. In addition, two channels correspond to polarization mixing: $\mathrm{TM} \leftrightarrows \mathrm{TE}$ and $\mathrm{TE} \leftrightarrows \mathrm{TM}$. Because of the symmetry of our geometry with identical spheres, the two polarization-mixing channels provide equal contributions and we will only consider their sum.

Figure 3 displays the temperature dependence of the three different contributions to the Casimir entropy scaled by the high-temperature Casimir entropy $\mathcal{S}_{\mathrm{HT}}^{\mathrm{P}}$ for perfectly conducting spheres. The parameters equal those used in Fig. 1 but no curves for the highest dc conductivity are shown.

In Fig. 3(a), the contribution of the TM channel is depicted. We only show the curve corresponding to $\gamma d / c=10^{2}$ but omit the curves for $\gamma d / c=10^{4}$ and for perfect conductors. According to Eqs. (6) and (8), the reflection coefficient for TM modes depends only very weakly on the dc conductivity and, in fact, the other curves would lie within not more than one line width. As discussed before, at high temperatures the TM channel contributes $4 / 5$ of the total Casimir entropy. The vertical axes in Fig. 3 are drawn to scale so that a

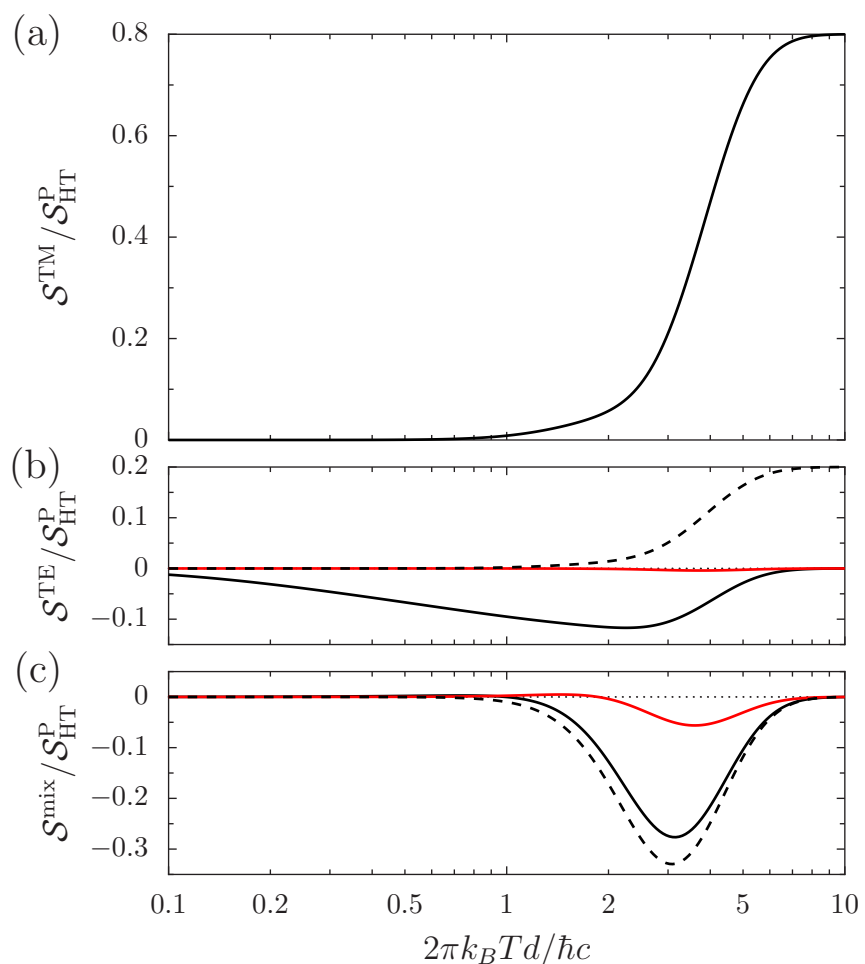

FIG. 3. (Color online) The contributions from (a) the TM channel, (b) the TE channel, and (c) the polarization-mixing channels to the Casimir entropy are shown as function of temperature for a sphere-sphere geometry with $d / R=20$. All contributions are scaled by the total high-temperature Casimir entropy $\mathcal{S}_{\mathrm{HT}}^{\mathrm{P}}$ for perfectly conducting spheres. The parameters are chosen as in Fig. 1, i.e., the dashed lines correspond to perfect metal spheres while the solid black and red lines correspond to Drude-type metal spheres with $\gamma d / c=10^{2}$ and $10^{4}$, respectively, and $\omega_{P} d / 2 \pi c=400$. For the sake of clarity, panel (a) displays only one line because all other lines deviate by not more than one line width. To facilitate the comparison between the contributions of the three types of scattering channels, the scales on the vertical axes are chosen to be equal.

comparison between the three contributions is facilitated. While the TM channel largely dominates at high temperatures, this is no longer the case at low temperatures, thus opening the possibility for negative Casimir entropies.

The temperature dependence of the TE channel shown in Fig. 3(b) displays the same features as the Casimir entropy in the plane-plane geometry depicted in Fig. 1(a) once the contribution arising from the TM modes is subtracted. We recognize the shift between the Casimir entropies for perfectly conducting spheres and Drude-type metal spheres at high temperatures due to the vanishing reflectivity for the TE modes at zero frequency. For not too small dc conductivity, i.e., the solid black curve, the contribution to the Casimir entropy becomes negative and goes to zero on a temperature scale proportional to $\gamma$. For bad conductors, i.e., the red solid curve, the contribution of the TE channel almost vanishes, in agreement with our discussion of the Rayleigh limit. The scattering channel analysis thus confirms that the TE modes are not only responsible for the dissipative contribution to the negative Casimir entropy in the plane-plane geometry, but also 


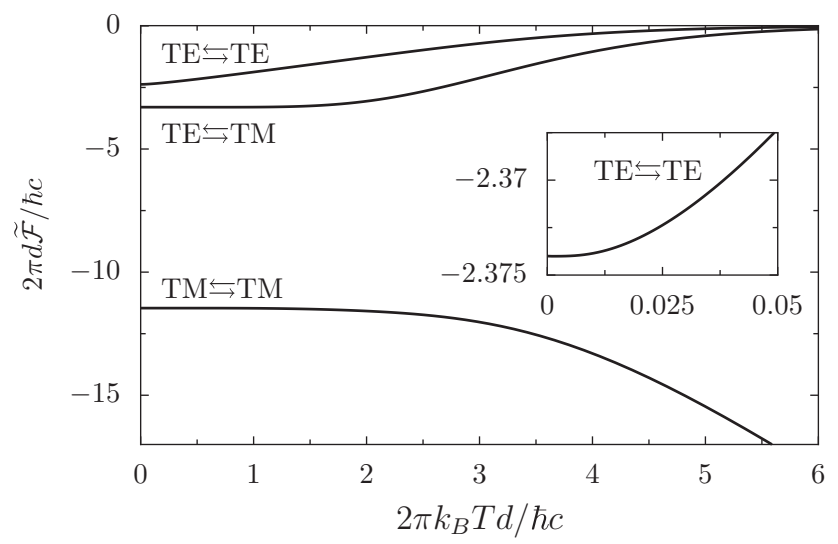

FIG. 4. The contributions of the different scattering channels to the scaled free energy $\widetilde{\mathcal{F}}=(d / R)^{6} \mathcal{F}$ are shown as a function of temperature. The distance between the two Drude-type metal spheres is given by $d / R=20$ and the material parameters are $\gamma d / c=10$ and $\omega_{P} d / 2 \pi c=400$. The inset displays the low-temperature behavior of the TE channel and demonstrates that the corresponding contribution to the Casimir entropy vanishes in the zero-temperature limit.

in the sphere-sphere geometry. It is worth mentioning that the same conclusion can be shown to hold true for the plane-sphere geometry.

Polarization-mixing channels do not exist in the planeplane geometry but contribute to the Casimir entropy in the plane-sphere and sphere-sphere geometries. The temperature dependence for the latter case is shown in Fig. 3(c). From Refs. [29] and [30] it is already known that the polarizationmixing channels are associated with the geometric origin of a negative Casimir entropy. Here, we can now study the dependence of this feature as a function of the Drude damping constant $\gamma$.

As the curves in Fig. 3(c) show, the negative Casimir entropy tends to be increasingly suppressed with increasing damping constant $\gamma$. In contrast to the TE channel in Fig. 3(b), a continuous transition exists from perfectly conducting spheres, where the negative Casimir entropy is most pronounced, to bad Drude-type metal spheres, where the mode mixing is significantly suppressed. Reducing the dc conductivity even further would ultimately result in a vanishing geometric contribution to a negative Casimir entropy [29,30].

We conclude that the geometric and dissipative contributions to a negative Casimir entropy can clearly be distinguished by means of a scattering-channel analysis provided the single round-trip condition is met, i.e., $d \gg R$. Nevertheless, the two types of channels share a common feature as can be seen from Fig. 4, where the contributions of the different scattering channels to the Casimir free energy is shown as a function of temperature.

The contributions of both TE and polarization-mixing channels vanish in the high-temperature limit. This behavior is in clear contrast to the usual behavior found for the TM channel, which yields a negative contribution decreasing linearly with temperature at high temperatures. In view of Eq. (1) and the fact that the contributions to the Casimir free energy at zero temperature are negative, the temperature dependence of the first two channels necessarily implies a negative contribution to the Casimir entropy in some temperature range.

This common feature should not obscure their different physical origins. For the TE channel, the contribution to the Casimir entropy at high temperatures vanishes because of the vanishing zero-frequency reflectivity. In contrast, for the polarization-mixing channel, no contribution to the Casimir entropy can occur at zero frequency where the fields corresponding to the two modes are either purely electric or purely magnetic in any of the two coordinate systems centered at each sphere. The difference in the physical mechanism manifests itself in Figs. 3(b) and 3(c), where we observe that the transition from a Drude-type metal to a perfect conductor occurs continuously for the polarization-mixing channel while it does not for the TE channel.

\section{SPHERE-SPHERE CASIMIR ENTROPY BEYOND THE DIPOLE APPROXIMATION}

So far, our discussion of the Casimir entropy has relied on the large-distance assumption $d \gg R$. Only then it suffices to consider single round-trips and the contributions of dissipative and geometric origin can be added. When the distance between the two spheres is decreased, it becomes necessary to go beyond the dipole approximation and to allow for scattering processes involving more than a single round-trip.

In this section, we first discuss the role of multiple roundtrip contributions for perfectly conducting spheres and thus concentrate on the geometrically induced negative Casimir entropy. In a second step, we add dissipation by considering Drude-type metal spheres.

Figure 5 shows the Casimir entropy as a function of the distance $d$ between two perfectly conducting spheres. The temperature is kept fixed at $2 \pi k_{B} T R / \hbar c=1$. The black points correspond to the full calculation based on Eq. (2), including multipoles up to $\ell_{\max }=60$ while the gray points

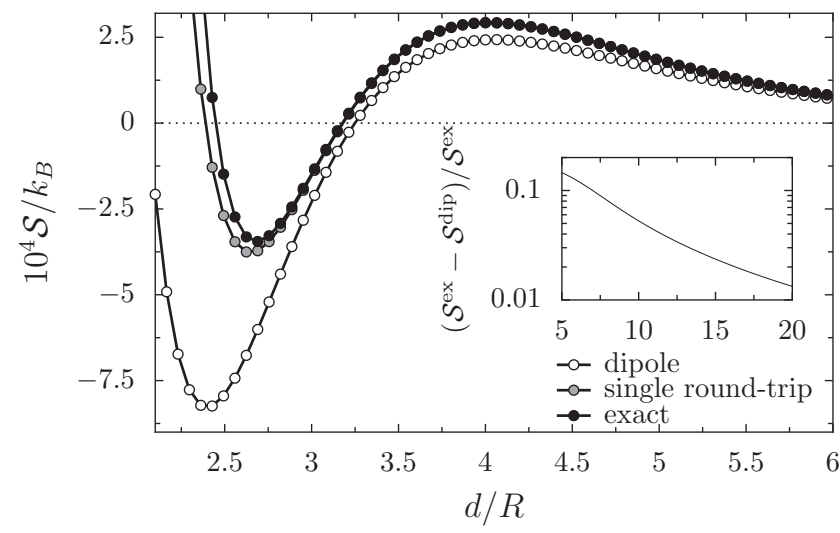

FIG. 5. The Casimir entropy $\mathcal{S}$ is presented as a function of the distance $d$ between two perfectly conducting spheres of radius $R$ at $2 \pi k_{B} T R / \hbar c=1$ for different levels of approximation. Results obtained within the single round-trip approximation are displayed as white and gray points. While the first ones correspond to the dipole approximation, the latter include higher multipoles up to $\ell_{\max }=60$. The numerically exact result depicted by black points is obtained on the basis of Eq. (2) with $\ell_{\max }=60$. The inset shows the relative difference of the dipole approximation $\mathcal{S}^{\text {dip }}$ and the exact result $\mathcal{S}^{\text {ex }}$. 


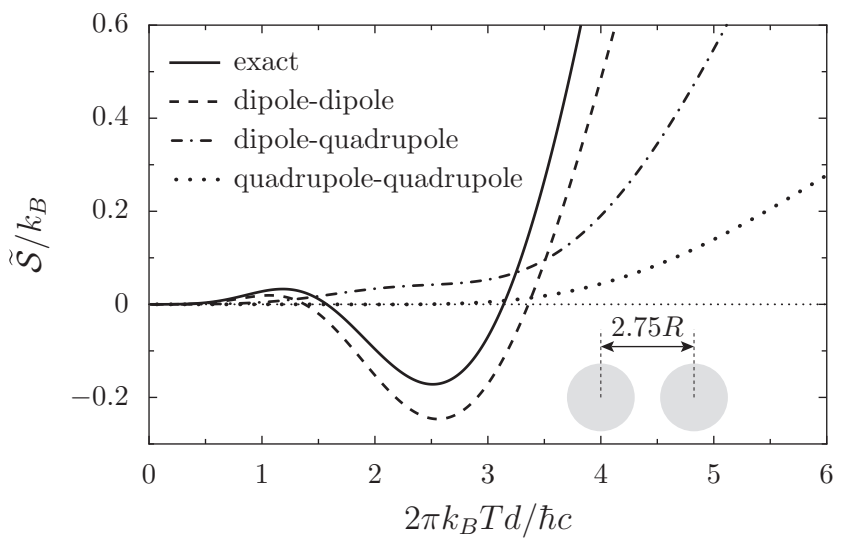

FIG. 6. The scaled Casimir entropy $\widetilde{\mathcal{S}}=(d / R)^{6} \mathcal{S}$ as a function of temperature is shown as solid line accounting for multipoles up to $\ell_{\max }=15$. The contributions arising from dipole-dipole, dipolequadrupole, and quadrupole-quadrupole scattering are depicted as dashed, dash-dotted, and dotted line, respectively. The distance between the perfectly conducting spheres is $d=2.75 R$.

correspond to the single round-trip approximation Eq. (4). The white points are obtained by additionally applying the dipole approximation.

The inset of Fig. 5 shows how the single round-trip dipole approximation approaches the exact result for the Casimir entropy as the distance between the two spheres is increased. At $d / R=20$, the distance chosen in the previous sections, the relative error reaches about one percent.

At the temperature chosen here, the Casimir entropy is positive at large distances between the two spheres. Decreasing the distance, the entropy will eventually become negative and go through a minimum before rising again to positive values [28]. Such a distance dependence was also found for the plane-sphere geometry [23,24]. Interestingly, even the single round-trip dipole approximation (white points) is capable of qualitatively describing this distance dependence of the Casimir entropy.

Including higher multipoles and multiple round-trips yields significant positive contributions to the Casimir entropy, in particular at relatively small distances between the two spheres. However, already the single round-trip approximation (gray points) provides a good description almost down to distances where the minimum of the Casimir entropy is reached. Only at even smaller distances multiple round-trip contributions become relevant (black points).

Figure 5 clearly demonstrates that for not too small distances $d$ the main correction to the single round-trip dipole approximation consists in the contribution of higher multipoles. We therefore analyze in Fig. 6 how the dipolequadrupole and quadrupole-quadrupole channels contribute to the temperature dependence of the Casimir entropy. The distance between the two perfectly conducting spheres is chosen as $d=2.75 R$, i.e., close to the minimum of the Casimir entropy in Fig. 5.

The solid line depicts the Casimir entropy, including multipoles up to $\ell_{\max }=15$, while the contributions due to dipole-dipole, dipole-quadrupole, and quadrupole-quadrupole scattering are displayed by the dashed, dash-dotted, and dotted

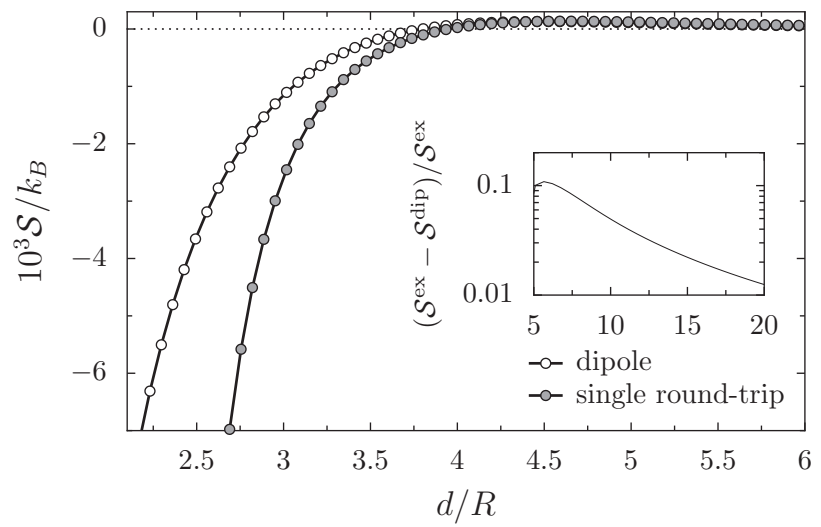

FIG. 7. The Casimir entropy $\mathcal{S}$ is presented as a function of the distance $d$ between Drude-type metal spheres of radius $R$ at $2 \pi k_{B} T R / \hbar c=1$ within the single round-trip approximation. The material parameters correspond to those of the best conductor represented by a blue curve in Fig. 1, i.e., $\omega_{P} R / 2 \pi c=20$ and $\omega_{P} / 2 \pi \gamma=4 \times 10^{4}$. The white points correspond to the dipole approximation while the gray points include higher multipoles up to $\ell_{\max }=60$. Numerically exact results accounting for multiple round-trips cannot be distinguished from the gray points in this figure and are therefore not shown. The inset displays the relative difference of the dipole approximation $\mathcal{S}^{\text {dip }}$ and the exact result $\mathcal{S}^{\text {ex }}$.

lines, respectively, and comprise all polarization channels. In agreement with Ref. [29] we find that the negative Casimir entropy is caused exclusively by dipole scattering. In fact, as can be seen from Fig. 6 for dipole-quadrupole and quadrupole-quadrupole scattering, higher multipoles lead to positive Casimir entropy contributions. Still, an analysis of the polarization channels involved in the multipole contributions depicted in Fig. 6 reveals that they have the same signs as the corresponding dipole-dipole contributions. However, for higher multipoles the negative contribution from the polarization-mixing channel is shifted to larger temperatures where the polarization-conserving channels dominate, yielding an overall result with positive sign.

We now turn to the dissipative case and consider Drudetype metal spheres. The distance dependence of the Casimir entropy is depicted in Fig. 7 for $2 \pi k_{B} T R / \hbar c=1$. The material parameters correspond to those of the best conductor considered in Sec. II and represented by the blue line in Fig. 1. Only results within the single round-trip approximation are displayed in Fig. 7, with white and gray points representing, respectively, the dipole approximation and the Casimir entropy with higher multipoles included. For the data presented here, the numerically exact result accounting for multiple roundtrips practically coincides with the gray points.

In contrast to perfectly conducting spheres, the contributions arising from higher multipoles are now negative and lead to a Casimir entropy increasing monotonically with distance. While the geometrically induced negative Casimir entropy is only due to dipole scattering, this is not the case for the negative Casimir entropy caused by dissipation. Here, higher multipole scattering also contributes with negative values. This important difference is to be expected because dissipation can give rise to a negative Casimir entropy even in the plane-plane geometry. 
As the inset in Fig. 7 shows, the deviation of the single round-trip dipole approximation from the numerically exact result is comparable for both perfect conductor spheres and Drude-type metal spheres. However, because of the missing high-temperature contribution of the TE modes, the absolute value of the Casimir entropy in the dissipative case exceeds the one for perfect conductors by one order of magnitude for the temperatures considered here. As a consequence, multiple round-trip corrections are much less important in Fig. 7 than they were in Fig. 5. However, in both cases, the approximations central to our analysis - single round-trip and dipole scattering-are well justified for the large distances $d / R=20$ chosen in the previous sections.

\section{CONCLUSIONS}

Dissipation and scattering geometry represent possible sources of negative Casimir entropies. While the first mechanism has been studied for quite some time in the plane-plane geometry, the second mechanism was identified for planesphere and sphere-sphere geometries with perfect conductors. For Drude-type metals of sufficiently large dc conductivity, we have shown the coexistence of both mechanisms by going beyond the Rayleigh limit.

In the large-distance limit, where only single round-trips are relevant, we employed a scattering-channel analysis to disentangle dissipative and geometric origins of the negative Casimir entropy. Concentrating on the sphere-sphere geometry, we found that dissipation acts via the TE channel as in the plane-plane geometry. In contrast, the geometric contribution can be traced back to the polarization-mixing channel as in the perfectly conducting case. Although being of different physical origin, both mechanisms are associated with a vanishing free energy in the high-temperature limit.

Focussing on the geometrical origin of negative Casimir entropies, we have found for perfectly conducting scatterers that while the dipole-dipole channel can lead to a negative contribution to the Casimir entropy, higher multipoles yield a positive contribution. With decreasing distance between the scatterers, the higher multipoles become dominant and the total Casimir entropy turns positive, thereby making connection with the plane-plane geometry for perfect conductors. In contrast, for the dissipative mechanism leading to negative Casimir entropies, the negative contribution is not restricted to dipole scattering.

\section{ACKNOWLEDGMENTS}

We are grateful to A. Lambrecht, S. Reynaud, A. CanaguierDurand, R. Guérout, and K. Milton for many insightful discussions. This work has been supported by the DAAD and CAPES through the PROBRAL program. In addition, PAMN acknowledges financial support by FAPERJ and CNPq (Brazilian agencies).

\section{APPENDIX: REFLECTION AND TRANSLATION OPERATORS}

For the reflection at a sphere, the scattering operator $\mathcal{R}$ occurring in Eq. (3) is naturally represented in the spherical multipole basis characterized by $\ell$ and $m$. The corresponding matrix elements are given by the Mie coefficients [40]. At imaginary frequencies and for nonmagnetic spheres, $\mu=1$, with refractive index $n=\sqrt{\varepsilon}$, they can be expressed as

$$
a_{\ell}=(-1)^{\ell+1} \frac{\pi}{2} \frac{n \psi_{\ell}(n k R) \psi_{\ell}^{\prime}(k R)-\psi_{\ell}(k R) \psi_{\ell}^{\prime}(n k R)}{n \psi_{\ell}(n k R) \xi_{\ell}^{\prime}(k R)-\xi_{\ell}(k R) \psi_{\ell}^{\prime}(n k R)}
$$

for TM polarization, and

$$
b_{\ell}=(-1)^{\ell+1} \frac{\pi}{2} \frac{\psi_{\ell}(n k R) \psi_{\ell}^{\prime}(k R)-n \psi_{\ell}(k R) \psi_{\ell}^{\prime}(n k R)}{\psi_{\ell}(n k R) \xi_{\ell}^{\prime}(k R)-n \xi_{\ell}(k R) \psi_{\ell}^{\prime}(n k R)}
$$

for TE polarization. The Riccati-Bessel functions are defined in terms of modified spherical Bessel functions of the first kind and second kind [45], as

$$
\psi_{\ell}(\rho)=\rho i_{\ell}(\rho), \quad \xi_{\ell}(\rho)=\rho k_{\ell}(\rho) .
$$

The spherical vector wave basis refers to a coordinate system with origin in the center of one of the spheres. A basis change from one center to another one can be done by means of translation formulas [46-49], which can also be expressed in terms of imaginary wave vectors [44]. The matrix elements of the translation operator take a relatively simple form if the translation occurs along the $z$ axis. Then, $m$ is conserved while $\ell$ and the polarization $P$ can change. For imaginary wave numbers $k$, the matrix elements can be cast into the form

$$
\begin{aligned}
\mathcal{T}_{\ell_{1}, \ell_{2} ; m}^{P P^{\prime}}(k d)= & \frac{(-1)^{m+1}( \pm \mathrm{i})^{\ell_{1}-\ell_{2}}}{\sqrt{\pi \ell_{1}\left(\ell_{1}+1\right) \ell_{2}\left(\ell_{2}+1\right)}} \\
& \times \sum_{\ell^{\prime}=\left|\ell_{1}-\ell_{2}\right|}^{\ell_{1}+\ell_{2}} c_{\ell_{1}, \ell_{2}, \ell^{\prime} ; m}^{P P^{\prime}} Y_{-m, m, 0}^{\ell_{1}, \ell_{2}, \ell^{\prime}} k_{\ell^{\prime}}(k d),
\end{aligned}
$$

where the initial and final polarizations $P$ and $P^{\prime}$, respectively, may be equal or different. The \pm sign has to be interpreted as positive if the translation occurs in the sense of the $z$ axis while the negative sign applies for translations in the opposite direction.

If the polarization is conserved, $P=P^{\prime}$, the coefficient appearing in Eq. (A4) reads

$c_{\ell_{1}, \ell_{2}, \ell^{\prime} ; m}^{P P}=2 \sqrt{2 \ell^{\prime}+1}\left[\ell_{1}\left(\ell_{1}+1\right)+\ell_{2}\left(\ell_{2}+1\right)-\ell^{\prime}\left(\ell^{\prime}+1\right)\right]$,

while for different polarizations we obtain

$$
c_{\ell_{1}, \ell_{2}, \ell^{\prime} ; m}^{P P^{\prime}}= \pm 4 \sqrt{\left(2 \ell^{\prime}+1\right)} m k d \quad\left(P \neq P^{\prime}\right) .
$$

In the latter case, the matrix elements of the translation operator vanish in the limit of vanishing wave number $k$ as well as for $m=0$.

The Gaunt coefficients (see, e.g., Ref. [50]),

$$
\begin{aligned}
Y_{-m, m, 0}^{\ell_{1}, \ell_{2}, \ell^{\prime}}= & \sqrt{\frac{\left(2 \ell_{1}+1\right)\left(2 \ell_{2}+1\right)\left(2 \ell^{\prime}+1\right)}{4 \pi}} \\
& \times\left(\begin{array}{ccc}
\ell_{1} & \ell_{2} & \ell^{\prime} \\
0 & 0 & 0
\end{array}\right)\left(\begin{array}{ccc}
\ell_{1} & \ell_{2} & \ell^{\prime} \\
-m & m & 0
\end{array}\right),
\end{aligned}
$$

do not require the explicit evaluation of the two Wigner $3 j$ symbols but can be determined efficiently by means of recurrence relations [51].

In the dipole limit, $\ell_{1}=\ell_{2}=1$, the matrix elements of the translation operator can be written as 


$$
\begin{gathered}
\mathcal{T}_{1,1 ; 0}^{P P} \mathcal{T}_{1,1 ; 0}^{P P}=9 e^{-2 k d}\left(\frac{1}{(k d)^{2}}+\frac{1}{(k d)^{3}}\right)^{2}, \\
\mathcal{T}_{1,1 ; 1}^{P P} \mathcal{T}_{1,1 ; 1}^{P P}=\frac{9}{4} e^{-2 k d}\left(\frac{1}{k d}+\frac{1}{(k d)^{2}}+\frac{1}{(k d)^{3}}\right)^{2},
\end{gathered}
$$

$$
\mathcal{T}_{1,1 ; 1}^{P P^{\prime}} \mathcal{T}_{1,1 ; 1}^{P^{\prime} P}=-\frac{9}{4} e^{-2 k d}\left(\frac{1}{k d}+\frac{1}{(k d)^{2}}\right)^{2} .
$$

These expressions make the suppression of large wave numbers mentioned in Sec. III explicit.
[1] P. Hänggi, G.-L. Ingold, and P. Talkner, Finite quantum dissipation: The challenge of obtaining specific heat, New J. Phys. 10, 115008 (2008).

[2] G.-L. Ingold, P. Hänggi, and P. Talkner, Specific heat anomalies of open quantum systems, Phys. Rev. E 79, 061105 (2009).

[3] G.-L. Ingold, Thermodynamic anomaly of the free damped quantum particle: The bath perspective, Eur. Phys. J. B 85, 30 (2012).

[4] B. Spreng, G.-L. Ingold, and U. Weiss, Reentrant classicality of a damped system, Europhys. Lett. 103, 60007 (2013).

[5] R. Adamietz, G.-L. Ingold, and U. Weiss, Thermodynamic anomalies in the presence of dissipation: From the free particle to the harmonic oscillator, Eur. Phys. J. B 87, 90 (2014).

[6] S. Florens and A. Rosch, Climbing the Entropy Barrier: Driving the Single- Towards the Multichannel Kondo Effect by a Weak Coulomb Blockade of the Leads, Phys. Rev. Lett. 92, 216601 (2004).

[7] R. Žitko and T. Pruschke, Anomaly in the heat capacity of Kondo superconductors, Phys. Rev. B 79, 012507 (2009).

[8] M. Campisi, P. Talkner, and P. Hänggi, Thermodynamics and fluctuation theorems for a strongly coupled open quantum system: An exactly solvable case, J. Phys. A: Math. Theor. 42, 392002 (2009).

[9] M. Campisi, D. Zueco, and P. Talkner, Thermodynamic anomalies in open quantum systems: Strong coupling effects in the isotropic XY model, Chem. Phys. 375, 187 (2010).

[10] A. Sulaiman, F. P. Zen, H. Alatas, and L. T. Handoko, Anharmonic oscillation effect on the Davydov-Scott monomer in a thermal bath, Phys. Rev. E 81, 061907 (2010).

[11] M. Boström and B. E. Sernelius, Thermal Effects on the Casimir Force in the 0.1-5 $\mu \mathrm{m}$ Range, Phys. Rev. Lett. 84, 4757 (2000).

[12] V. B. Bezerra, G. L. Klimchitskaya, and V. M. Mostepanenko, Correlation of energy and free energy for the thermal Casimir force between real metals, Phys. Rev. A 66, 062112 (2002).

[13] M. Boström and B. E. Sernelius, Entropy of the Casimir effect between real metal plates, Physica A 339, 53 (2004).

[14] V. B. Bezerra, G. L. Klimchitskaya, V. M. Mostepanenko, and C. Romero, Violation of the Nernst heat theorem in the theory of the thermal Casimir force between Drude metals, Phys. Rev. A 69, 022119 (2004).

[15] I. Brevik, J. B. Aarseth, J. S. Høye, and K. A. Milton, Temperature dependence of the Casimir force for metals, in Proceedings of the Sixth Workshop on Quantum Field Theory Under the Influence of External Conditions, edited by K. A. Milton (Rinton Press, Princeton, 2004), pp. 54-65.

[16] I. Brevik, J. B. Aarseth, J. S. Høye, and K. A. Milton, Temperature dependence of the Casimir effect, Phys. Rev. E 71, 056101 (2005).

[17] V. B. Svetovoy and R. Esquivel, Nonlocal impedances and the Casimir entropy at low temperatures, Phys. Rev. E 72, 036113 (2005).
[18] V. B. Svetovoy and R. Esquivel, The Casimir free energy in high- and low-temperature limits, J. Phys. A: Math. Gen. 39, 6777 (2006).

[19] V. B. Bezerra, G. L. Klimchitskaya, V. M. Mostepanenko, and C. Romero, Lifshitz theory of atom-wall interaction with applications to quantum reflection, Phys. Rev. A 78, 042901 (2008).

[20] S. Å. Ellingsen, I. Brevik, J. S. Høye, and K. A. Milton, Low temperature Casimir-Lifshitz free energy and entropy: The case of poor conductors, J. Phys.: Conf. Ser. 161, 012010 (2009).

[21] G.-L. Ingold, A. Lambrecht, and S. Reynaud, Quantum dissipative Brownian motion and the Casimir effect, Phys. Rev. E 80, 041113 (2009).

[22] L. P. Pitaevskii, Casimir-Lifshitz Forces and Entropy, in Quantum Field Theory Under the Influence of External Conditions (QFEXT09), edited by K. A. Milton and M. Bordag (World Scientific, Singapore, 2010), p. 227.

[23] A. Canaguier-Durand, P. A. Maia Neto, A. Lambrecht, and S. Reynaud, Thermal Casimir Effect in the Plane-Sphere Geometry, Phys. Rev. Lett. 104, 040403 (2010).

[24] A. Canaguier-Durand, P. A. Maia Neto, A. Lambrecht, and S. Reynaud, Thermal Casimir effect for Drude metals in the planesphere geometry, Phys. Rev. A 82, 012511 (2010).

[25] M. Bordag and I. G. Pirozhenko, Casimir entropy for a ball in front of a plane, Phys. Rev. D 82, 125016 (2010).

[26] R. Zandi, T. Emig, and U. Mohideen, Quantum and thermal Casimir interaction between a sphere and a plate: Comparison of Drude and plasma models, Phys. Rev. B 81, 195423 (2010).

[27] A. Weber and H. Gies, Nonmonotonic thermal Casimir force from geometry-temperature interplay, Phys. Rev. D 82, 125019 (2010).

[28] P. Rodriguez-Lopez, Casimir energy and entropy in the spheresphere geometry, Phys. Rev. B 84, 075431 (2011).

[29] G.-L. Ingold, S. Umrath, M. Hartmann, R. Guérout, A. Lambrecht, S. Reynaud, and K. A. Milton, Geometric origin of negative Casimir entropies: A scattering-channel analysis, Phys. Rev. E 91, 033203 (2015).

[30] K. A. Milton, R. Guérout, G.-L. Ingold, A. Lambrecht, and S. Reynaud, Negative Casimir Entropies in nanoparticle interactions, J. Phys.: Condens. Matter 27, 214003 (2015).

[31] R. S. Decca, D. López, E. Fischbach, G. L. Klimchitskaya, D. E. Krause, and V. M. Mostepanenko, Test of new physics from precise measurements of the Casimir pressure between gold-coated plates, Phys. Rev. D 75, 077101 (2007).

[32] A. A. Banishev, G. L. Klimchitskaya, V. M. Mostepanenko, and U. Mohideen, Casimir interaction between two magnetic metals in comparison with nonmagnetic test bodies, Phys. Rev. B 88, 155410 (2013).

[33] A. O. Sushkov, W. J. Kim, D. A. R. Dalvit, and S. K. Lamoreaux, Observation of the thermal Casimir force, Nat. Phys. 7, 230 (2011). 
[34] D. Garcia-Sanchez, K. Y. Fong, H. Bhaskaran, S. Lamoreaux, and H. X. Tang, Casimir Force and in situ Surface Potential Measurements on Nanomembranes, Phys. Rev. Lett. 109, 027202 (2012).

[35] E. M. Lifshitz and L. P. Pitaevskiı̌, Statistical Physics, Part 2, sect. 81 (Pergamon Press, Oxford, UK, 1980).

[36] R. Guérout, A. Lambrecht, K. A. Milton, and S. Reynaud, Derivation of the Lifshitz-Matsubara sum formula for the Casimir pressure between metallic plane mirrors, Phys. Rev. E 90, 042125 (2014).

[37] G. Jourdan, A. Lambrecht, F. Comin, and J. Chevrier, Quantitative non-contact dynamic Casimir force measurements, Europhys. Lett. 85, 31001 (2009).

[38] G. Torricelli, I. Pirozhenko, S. Thornton, A. Lambrecht, and C. Binns, Casimir force between a metal and a semimetal, Europhys. Lett. 93, 51001 (2011).

[39] M. Masuda and M. Sasaki, Limits on Nonstandard Forces in the Submicrometer Range, Phys. Rev. Lett. 102, 171101 (2009).

[40] C. F. Bohren and D. R. Huffman, Absorption and Scattering of Light by Small Particles (Wiley-VCH, Weinheim, Germany, 2007).

[41] M. T. Jaekel and S. Reynaud, Casimir force between partially transmitting mirrors, J. Phys. I France 1, 1395 (1991).
[42] C. Genet, A. Lambrecht, and S. Reynaud, Casimir force and the quantum theory of lossy optical cavities, Phys. Rev. A 67, 043811 (2003).

[43] A. Lambrecht, P. A. Maia Neto, and S. Reynaud, The Casimir effect within scattering theory, New J. Phys. 8, 243 (2006).

[44] T. Emig, N. Graham, R. L. Jaffe, and M. Kardar, Casimir Forces Between Arbitrary Compact Objects, Phys. Rev. Lett. 99, 170403 (2007).

[45] NIST Digital Library of Mathematical Functions. http://dlmf.nist.gov/10.25, Release 1.0.9 of 2014-08-29.

[46] S. Stein, Addition theorems for spherical wave functions, Quart. Appl. Math. 19, 15 (1961)

[47] O. R. Cruzan, Translational addition theorems for spherical vector wave functions, Quart. Appl. Math. 20, 33 (1962).

[48] J. H. Bruning and Y. T. Lo, Multiple Scattering of EM Waves by Spheres, Part I-Multipole expansion and ray-optical solution, IEEE Trans. Ann. Prop. 19, 378 (1971).

[49] R. C. Wittmann, Spherical wave operators and the translation formulas, IEEE Trans. Antennas. Propag. 36, 1078 (1988).

[50] J. Rasch and A. C. H. Yu, Efficient storage scheme for precalculated Wigner $3 j, 6 j$, and Gaunt coefficients, SIAM J. Sci. Comput. 25, 1416 (2004).

[51] Y.-L. Xu, Fast evaluation of Gaunt coefficients: Recursive approach, J. Comp. Appl. Math. 85, 53 (1997). 